\title{
The clinical spectrum and genetic variability of limb-girdle muscular dystrophy in a cohort of Chinese patients
}

\author{
Liang Wang ${ }^{1}$, Victor Wei Zhang ${ }^{2,3}$, Shaoyuan Li ${ }^{3}$, Huan Li ${ }^{1}$, Yiming Sun ${ }^{4}$, Jing Li $i^{1}$, Yuling Zhu', Ruojie He${ }^{1}$, \\ Jinfu Lin ${ }^{1}$ and Cheng Zhang ${ }^{1 *}$ (D)
}

\begin{abstract}
Background: Limb-girdle muscular dystrophy (LGMD) is a commonly diagnosed hereditary muscular disorder, characterized by the progressive weakness of the limb-girdle muscles. Although the condition has been wellcharacterized, clinical and genetic heterogeneity can be observed in patients with LGMD. Here, we aimed to describe the clinical manifestations and genetic variability among a cohort of patients with LGMD in South China.

Results: We analyzed the clinical information, muscle magnetic resonance imaging (MRI) findings, and genetic results obtained from 30 patients (24 families) with clinically suspected LGMD. In 24 probands, 38 variants were found in total, of which 18 were shown to be novel. Among the 30 patients, the most common subtypes were dysferlinopathy in eight (26.67\%), sarcoglycanopathies in eight [26.67\%; LGMD 2C in three (10.00\%), LGMD 2D in three (10.00\%), and LGMD 2F in two (6.67\%)], LGMD 2A in seven (23.33\%), followed by LGMD 1B in three (10.00\%), LGMD 2 I in three (10.00\%), and early onset recessive Emery-Dreifuss-like phenotype without cardiomyopathy in one (3.33\%). Furthermore, we also observed novel clinical presentations for LGMD 1B, 2F, and 2I patients with hypermobility of the joints in the upper limbs, a LGMD $2 F$ patient with delayed language development, and other manifestations. Moreover, distinct distributions of fatty infiltration in patients with LGMD 2A, dysferlinopathy, and the early onset recessive Emery-Dreifuss-like phenotype without cardiomyopathy were also observed based on muscle MRI results.
\end{abstract}

Conclusions: In this study, we expanded the clinical spectrum and genetic variability found in patients with LGMD, which provided additional insights into genotype and phenotype correlations in this disease.

Keywords: Limb-girdle muscular dystrophy, Clinical manifestation, Muscle magnetic resonance imaging, Molecular diagnosis, South China

\section{Background}

Limb-girdle muscular dystrophy (LGMD) is a commonly diagnosed hereditary muscular disorder, characterized by progressive weakness of the limb-girdle muscles $[1,2]$. As a group of neurogenetic diseases, LGMD is the fourth most common muscular dystrophy, with a pooled prevalence of 1.63 per 100,000 people, following myotonic dystrophy, dystrophinopathy, and facioscapulohumeral dystrophy [1-4]. The prevalence varies among

\footnotetext{
* Correspondence: zhangch6@mail.sysu.edu.cn

'Department of Neurology, National Key Clinical Department and Key

Discipline of Neurology, The First Affiliated Hospital, Sun Yat-sen University,

58 Zhongshan 2 Road, Guangzhou 510080, GD, China

Full list of author information is available at the end of the article
}

subtypes and regions. For example, the most common subtypes in Italy are LGMD 2A and 2B, while LGMD 2I is most common in Denmark [5, 6]. LGMD was first described by Erb and Leyden-Möbius in the late eighteenth century, and 30 distinct subtypes have been identified thus far [7]. On the basis of the origin of muscle weakness, LGMD was initially divided into the Erb phenotype (scapulohumeral type), Leyden-Möbius phenotype (pelvic girdle type), and Miyoshi phenotype (exceptionally distal type) [8]. Later, classifications were made based on the mode of inheritance; thus, LGMD patients can be subdivided into two main classes: LGMD subtype 1 with autosomal dominant inheritance and LGMD subtype 2

(c) The Author(s). 2018 Open Access This article is distributed under the terms of the Creative Commons Attribution 4.0 International License (http://creativecommons.org/licenses/by/4.0/), which permits unrestricted use, distribution, and reproduction in any medium, provided you give appropriate credit to the original author(s) and the source, provide a link to the Creative Commons license, and indicate if changes were made. The Creative Commons Public Domain Dedication waiver (http://creativecommons.org/publicdomain/zero/1.0/) applies to the data made available in this article, unless otherwise stated. 
with autosomal recessive inheritance [9]. LGMD subtype $2(2 \mathrm{~A}-2 \mathrm{U})$ is more common than LGMD subtype 1 $(1 \mathrm{~A}-1 \mathrm{H})$, and most of these subtypes have been shown to be associated with defects in specific genes [7].

LGMD is mainly described as progressive weakness and atrophy of the hip, shoulder, and proximal extremity muscles, with onset in the second decade of life [9]. Considering the genetic and phenotypic heterogeneity of this disease, LGMD should be considered in almost all undiagnosed patients complaining of primary muscle weakness [10]. Certain clinical features of LGMD subtypes can serve as valuable diagnostic clues, such as extremely high levels of serum creatine kinase $(\mathrm{CK})$ in LGMD 2B-F and LGMD 2I patients [11]. Other approaches such as muscle biopsy and muscle magnetic resonance imaging (MRI) may also help establish the clinical diagnosis [12, 13]. Moreover, genetic testing can provide accurate information for unambiguously establishing the genetic diagnosis, and it is currently available at a very low cost owing to the rapid development of sequencing techniques $[14,15]$. Therefore, a comprehensive understanding of the genetic variability and clinical spectrum of LGMD is essential before precise diagnosis, treatment and reproductive counselling can be offered [2].

Previously, clinical characteristics and the underlying molecular defects have been documented in patients with LGMD $[5,16,17]$. Here, we aimed to extend our knowledge and understanding of this disease to determine novel and important characteristics and genetic variants in patients with LGMD.

\section{Methods}

\section{Participants and patient information}

Thirty patients suspected to have LGMD were enrolled in this study from 2015 to 2017 from the Hereditary Neurological Disease Clinics of The First Affiliated Hospital, Sun Yat-sen University. The basic data obtained from these patients were recorded, including clinical histories and motor functions, and were evaluated by two experienced neurologists. Ages are presented as mean \pm standard deviation. The activity of $\mathrm{CK}$ was examined in each patient. Furthermore, we performed electromyography (EMG), cardiac function estimation, muscle tissue histology, immunohistochemical staining (IHC), and MRI according to previously published protocols $[18,19]$.

\section{Muscle histology}

Muscle biopsy specimens were obtained from the biceps brachii or quadriceps femoris. Biopsied muscles were frozen in isopentane chilled by liquid nitrogen. IHC staining was performed on $6 \mu \mathrm{m}$ frozen muscle sections according to the established protocol [20]. Antibodies against dystrophin, $\alpha$-sarcoglycan, $\beta$-sarcoglycan, $\gamma$-sarcoglycan, and $\delta$-sarcoglycan were purchased from Novocastra (Newcastle,
UK), while the anti-dysferlin antibody was purchased from Millipore (Darmstadt, Germany).

\section{Next-generation sequencing}

Blood samples $(2 \mathrm{~mL})$ from each patient were collected in EDTA-coated tubes. DNA $(3 \mu \mathrm{g})$ was extracted from these samples using the QIAamp DNA Blood Midi kit (Qiagen, Hilden, Germany). A next-generation sequencing (NGS) neuromuscular disorder panel was used to detect variants. The detailed NGS protocol and the list of genes detected using this panel are provided in Additional file 1. Briefly, the process included the construction of a genomic DNA library, capturing the locations of target genes, gene sequencing using an analytical platform (HiSeq 2000 system; Illumina, USA), and bioinformatics analysis. After the identification of the variants in the proband, family co-segregation analyses were performed using Sanger sequencing.

\section{Bioinformatics analysis}

Gene frequencies were determined using the $1000 \mathrm{Ge}$ nomes Project and ExAC [21, 22], and computational analysis of variants was performed using PolyPhen-2, SIFT, and MutationTaster [23-25]. The Human Gene Mutation Database, Leiden Open Variation Database, ClinVar, and Google Scholar were used to identify the reported variants [26-29]. The pathogenicity of the variants was estimated using the American College of Medical Genetics and Genomics (ACMG) guidelines [30].

\section{Results}

\section{Participants}

Detailed data were collected from 24 probands (Table 1). There were, in total, 30 patients in 24 families. Proband ages were between 3 and 44 years. There were 14 female probands $[58.33 \%$ of probands; 18 female patients (60.00\% of patients)] and 10 male probands [41.67\% of probands; 12 male patients $(40.00 \%$ of patients)]. Autosomal recessive types (LGMD 2) were most common [21 probands $(87.50 \%$ of probands) and 27 patients $(90.00 \%$ of patients)].

The most frequent subtypes were dysferlinopathy [seven probands (29.17\% of probands), eight patients (26.67\% of patients)], sarcoglycanopathies [LGMD 2C-F; seven probands $(29.17 \%$ of probands), eight patients (26.67\% of patients)], LGMD 2A [four probands $(16.67 \%$ of probands), seven patients (23.33\% of patients)], and LGMD 1B [three probands (12.50\% of probands); three patients $(10.00 \%$ of patients)]. Within sarcoglycanopathies, the frequencies of LGMD 2C, $2 \mathrm{D}$, and $2 \mathrm{~F}$ were three probands $[12.50 \%$ of probands; three patients (10.00\% of patients)], two probands [ $8.33 \%$ of probands; three patients (10.00\% of patients)], and two probands [ $8.33 \%$ of probands; two patients ( $6.67 \%$ of patients)], 


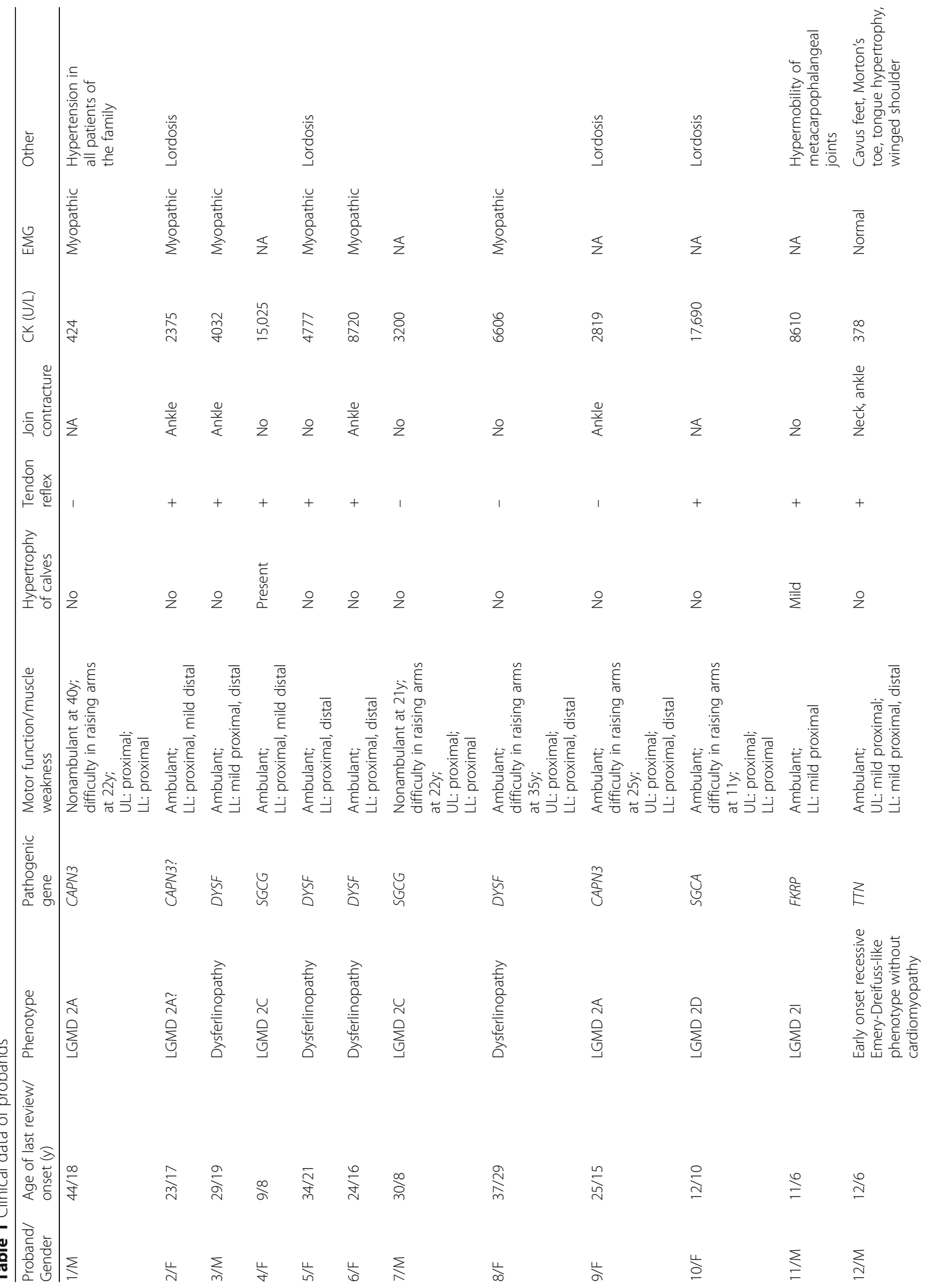




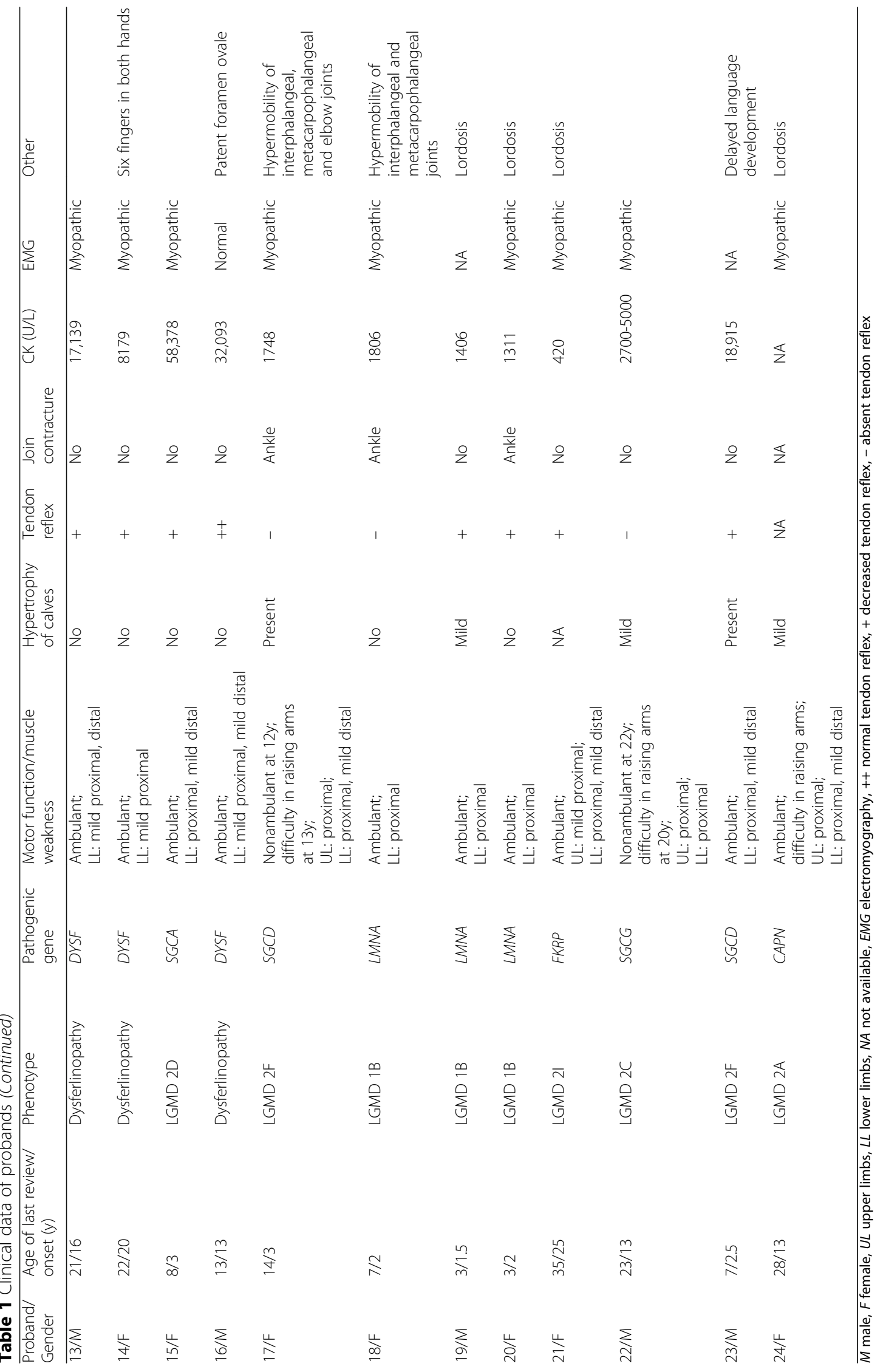


respectively. The remaining patients were affected by LGMD 2I [two probands (8.33\% of probands), three patients $(10.00 \%$ of patients)], except for a patient with the early onset recessive Emery-Dreifuss-like phenotype without cardiomyopathy. Because the disease history of the proband in each family could be collected accurately, the main results in this study represent the data of 24 probands, as below.

\section{Age of onset}

The subtype with the earliest age of onset was LGMD $1 \mathrm{~B}$ (1.83 \pm 0.29 years), followed by LGMD $2 \mathrm{~F}$ ( 2.5 and 3 years). Overall, the age of onset of sarcoglycanopathies was early, since most of them presented with muscle weakness no later than 10 years $(6.79 \pm 4.06$ years $)$. In addition, most probands with LGMD 2A and dysferlinopathy presented with muscle weakness during the second decade of life, wherein the ages of onset in LGMD $2 \mathrm{~A}$ and dysferlinopathy were $15.75 \pm 2.22$ years and $19.14 \pm 5.15$ years, respectively. Proband 12 had the early onset recessive Emery-Dreifuss-like phenotype without cardiomyopathy and complained of muscle weakness since the age of 6 years. Moreover, the difference of age of onset between two probands with LGMD 2I was large (6 and 25 years).

\section{Muscle weakness and motor function}

All probands had weakness of the proximal lower limbs to different degrees. In 10 probands (41.67\%), the proximal upper limbs were involved, among whom nine probands (37.50\%) had predominant weakness of both proximal upper limbs and proximal lower limbs. Furthermore, 15 probands (62.50\%) complained of weakness of the distal lower limbs, among whom eight probands had mild symptoms. When analyzing predominant involvements of proximal or distal limbs, the results indicated that most probands were characterized by predominant weakness of the scapular and/or pelvic muscles (83.33\%); however, three probands (proband 3 and 13 with dysferlinopathy and proband 12 with the early onset recessive Emery-Dreifuss-like phenotype without cardiomyopathy) were characterized by predominant weakness of the distal lower limbs and one proband, with dysferlinopathy (proband 16) characterized by mild weakness of both proximal and distal lower limbs.

Four probands (16.67\%) lost the ability of ambulation, among whom three had sarcoglycanopathies and one had LGMD 2A. Of 20 probands with the ability of ambulation, eight presented with an obvious waddling gait. When we evaluated probands' daily movements, we found that the number of probands losing the ability of jumping, climbing, and standing up from the ground were 10 (LGMD 2A: two; dysferlinopathy: three; sarcoglycanopathies: two; LGMD 1B: two; LGMD 2I: one), one (dysferlinopathy: one), and four (LGMD 2A, dysferlinopathy, LGMD 2D, and LGMD 2I, one each), respectively, in the probands with the ability of ambulation. Furthermore, four probands could jump normally (dysferlinopathy: two; LGMD 2I: one; early onset recessive Emery-Dreifuss-like phenotype without cardiomyopathy: one). A proband with dysferlinopathy could climb normally, and two probands with dysferlinopathy could stand up from the ground normally. The remaining probands had difficulties in these movements to different degrees. In addition, eight probands (33.33\%) had difficulties in raising arms, among whom four, three, and one had sarcoglycanopathies, LGMD 2A, and dysferlinopathy, respectively.

\section{Disease course}

All probands presented with progressive deterioration of muscle weakness. The mean time between disease onset and last review was $7.79 \pm 6.43$ years. Among four probands losing ambulation, the mean time between loss of ambulation and onset of disease for patients with sarcoglycanopathies was 10.00 years, and proband 1 with LGMD 2A lost the ability of ambulation at the age of 40 years, i.e., 22 years after the onset of weakness. The time between difficulty in raising arms and onset of disease for probands with sarcoglycanopathies varied from 1 year to 14 years (mean, 8 years), and the time for probands 1 and 9 with LGMD 2A and proband 8 with dysferlinopathy were 4,10 , and 6 years, respectively. The onset age of raising arms with difficulty in proband 24 with LGMD 2A was not available.

\section{Rare clinical features}

In this cohort, three probands had hypermobility of the joints. Proband 11 with LGMD 2I presented with hypermobility of the metacarpophalangeal joints (Fig. 1a), and proband 17 with LGMD 2F presented with hypermobility of the interphalangeal, metacarpophalangeal, and elbow joints. Furthermore, proband 18 with LGMD 1B presented with hypermobility of the interphalangeal and metacarpophalangeal joints.

In addition, proband 14 with dysferlinopathy had six fingers in both hands and proband 16 with dysferlinopathy had patent foramen ovale in his heart. Proband 23 with LGMD $2 \mathrm{~F}$ also demonstrated delayed language development. He could speak only three to five simple words at the age of 3 years, and language learning was slower compared to normal children. Language ability ameliorated gradually, and he could understand orders and have a conversation with other people at the age of 7 years.

Proband 12 presented a rare phenotype of early onset recessive Emery-Dreifuss-like phenotype without cardiomyopathy; thus, we describe his disease history in detail. 


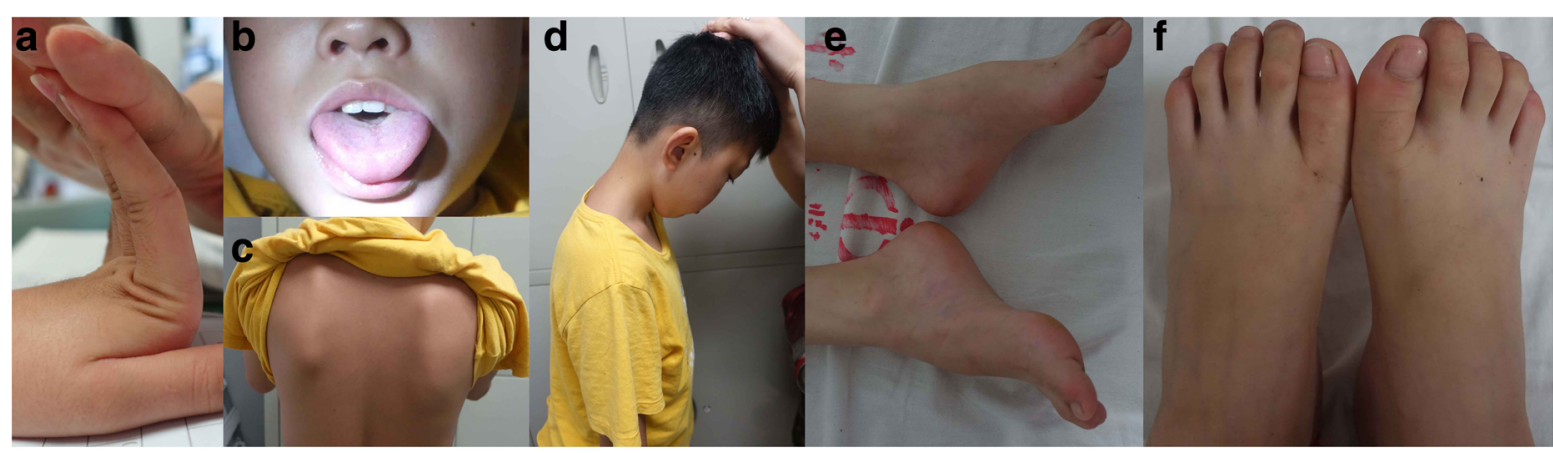

T1 weighted image

T2 weighted image

Fat suppression $\mathrm{T} 2$ weighted image

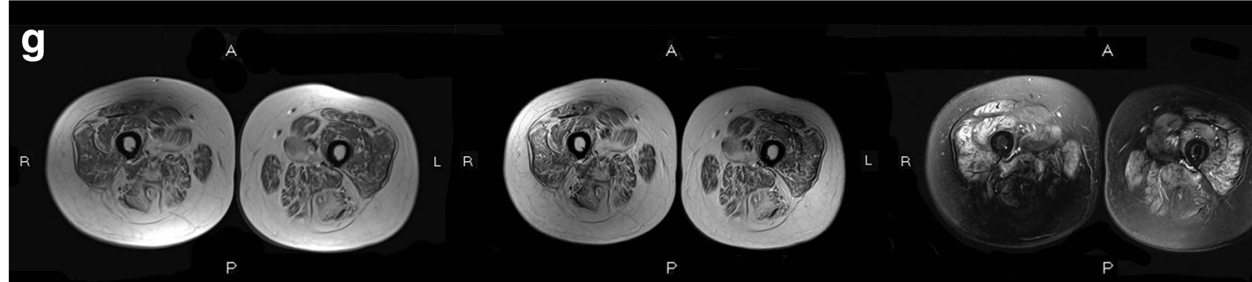

h

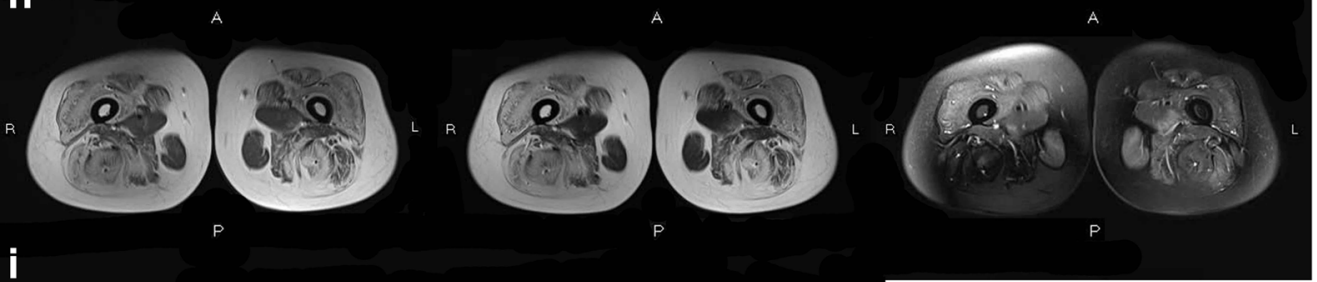

i

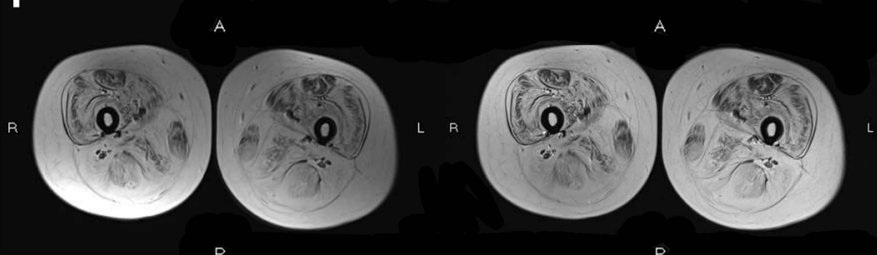

j
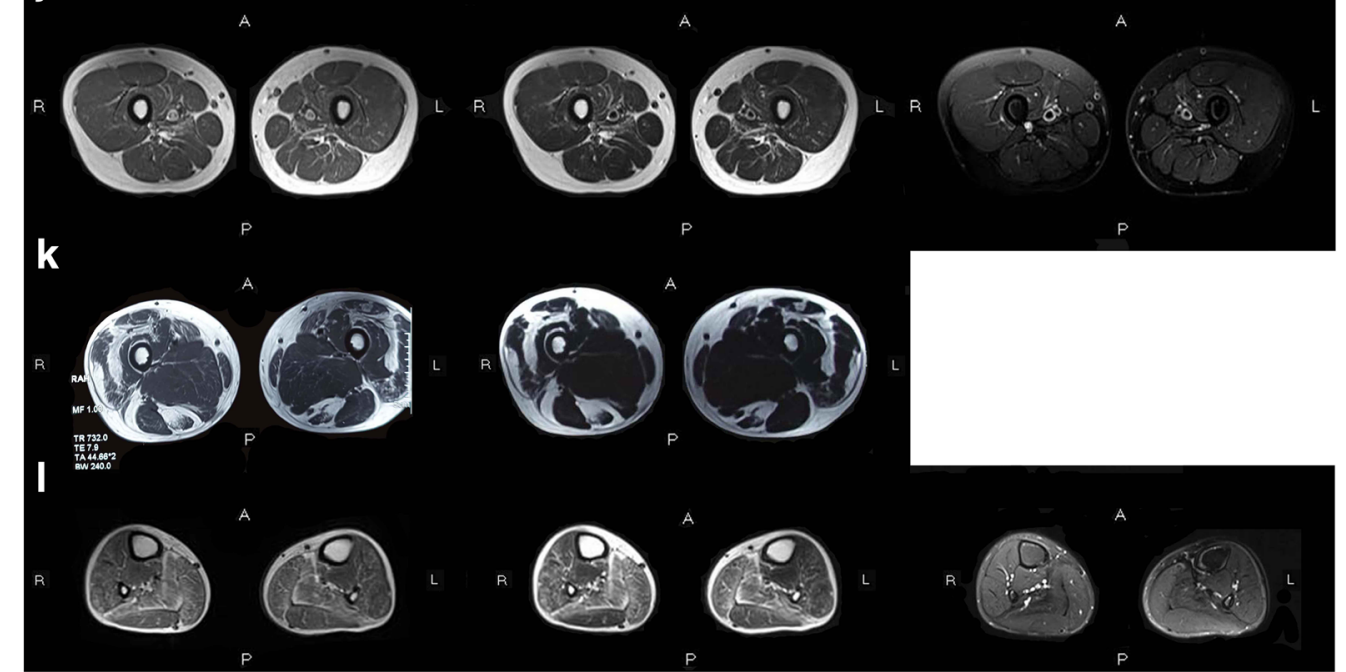

Fig. 1 (See legend on next page.) 
(See figure on previous page.)

Fig. 1 Clinical and magnetic resonance imaging (MRI) results obtained for all patients included in the study. (a) Hypermobility of the

metacarpophalangeal joints observed in proband 11. (b-e) Physical characteristics of proband 12. (b) Tongue hypertrophy. (c) Winged shoulder. (d) Neck contracture. (e) Ankle contracture and cavus feet. (f) Morton's toe. (g-I) Muscle MRI results obtained for probands 3, 5, 9, 11, and 12. T1weighted image, T2-weighted image, and fat-suppression T2-weighted image (left to right). MRI results of both thighs obtained for proband 3 (g), proband $5(\mathbf{h})$, proband 9 (i), proband 11 (j), and proband $12(\mathbf{k})$. (I) MRI results of both calves obtained for proband 12

He complained of mild difficulties when climbing and standing up from the ground since the age of 6 years, and muscle weakness progressed slowly with time. He was admitted to our clinic at the age of 12 years, with mild muscle weakness. Compared with that observed in the proximal limbs, dorsiflexion of the ankle muscles was obviously weaker, and he could not complete the sit-up movement. Facial, bulbar, and oculomotor muscles were not affected. Tongue hypertrophy (Fig. 1b) and winged shoulder (Fig. 1c) could be observed. His neck could not flex normally (Fig. 1d) and ankle could not dorsiflex fully (Fig. 1e) because of contractures. Moreover, some malformations of the feet were observed, such as cavus feet (Fig. 1e) and Morton's toe (the second toe was longer than the big toe, Fig. 1f) in both feet.

\section{CK levels, EMG, muscle histology, and cardiac examinations}

In 23 probands who underwent serum enzyme tests, CK levels (reference range: 25-200 U/L) were elevated. For the majority (69.57\%), the levels reached more than tenfold over the upper normal limit (ULN). Among probands (26.09\%) whose CK levels were more than 50-fold higher than the ULN, four demonstrated sarcoglycanopathies and two, dysferlinopathy. Furthermore, the CK levels of all probands with LGMD $1 \mathrm{~B}$ varied between fivefold and tenfold over the ULN. Among three probands whose CK levels were less than fivefold higher than the ULN, one each had advanced LGMD 2A (proband 1), the early onset recessive Emery-Dreifuss-like phenotype without cardiomyopathy (proband 12), and LGMD 2I (proband 21). Furthermore, all probands who underwent EMG except for probands 12 and 16 exhibited myopathic injuries (Table 1).

Nine probands underwent cardiac examinations. The cardiac examination results, including electrocardiography and ultrasonic cardiography, of probands 3, 11, $12,15,18$, and 21 were within normal limits. The remaining results indicated cardiac injuries. Mild mitral regurgitation was observed in proband 14, and patent foramen ovale, mild left atrial and ventricular enlargement were observed in proband 16. Besides, left ventricular high voltage was observed in proband 17.

Eight probands (probands 2, 3, 10, 11, 13, 14, 23, and 24) underwent muscle biopsy and histology analysis. Typical muscular dystrophy phenotypes were observed in all patients, including degeneration, regeneration, centralized myofiber nuclei, and different levels of connective tissue hyperplasia. Among these eight probands, muscle sections of probands 2 and 10 were stained with antibodies against dystrophin, dysferlin, $\alpha$-sarcoglycan, $\beta$-sarcoglycan, $\gamma$-sarcoglycan, and $\delta$-sarcoglycan. Although the muscle sections of proband 2 were not found to have any negative staining, IHC analysis results obtained for proband 10 were negative for sarcoglycan- $\alpha$ and sarcoglycan- $\beta$ staining, indicating sarcoglycanopathies. Muscle sections of probands 11, 13, and 23 were only stained with anti-dystrophin, and the results indicated positive staining. The remaining muscle samples were not stained using IHC.

\section{Muscle MRI}

MRI scans of the thigh were performed in probands 3, 5, $9,10,11,12$, and 15, and calf scans were performed in probands 12 and 13. The scan results of proband 3 (Fig. $1 \mathrm{~g}$ ) indicated fatty infiltration to various degrees in each muscle of both thighs, particularly in the adductor longus and posterior group of muscles (semitendinosus, semimembranosus, and biceps femoris). The involvement of the gracilis, sartorius, and quadriceps femoris was milder. Interestingly, the analysis of muscle edema indicated an opposite feature in the distribution of the involved muscles. The edema of the adductor longus and posterior group of muscles was milder than that of other muscles.

Obvious fatty infiltration of the thigh muscles was also observed in proband 5 (Fig. 1h); however, the adductor longus and gracilis were spared. Furthermore, fatty infiltration of the adductor magnus was relatively milder. In comparison to selective involvement of fatty infiltration, edema was more extensive in the thigh muscles. Similarly, fatty infiltration of the thigh muscles was also extensive in proband 9 (Fig. 1i). Obvious fatty infiltration was exhibited in almost all muscles. The involvement of the rectus femoris was relatively milder.

The sartorius, gracilis, and semitendinosus were spared in proband 10, and other muscles of the thighs represented different degrees of fatty infiltration. As for edema condition, the majority of the thigh muscles represented edema, except the sartorius, gracilis, semitendinosus, and adductor longus. In contrast, the fatty infiltration and edema of the thigh muscles were very mild in proband 11 (Fig. 1j). Mild patch-like fatty infiltration could be seen only in the adductor magnus. 
The selective involvement of fatty infiltration in proband 12 was noteworthy. In the thigh muscles (Fig. 1k), the following muscles were spared from fatty infiltration: the adductor magnus, adductor longus, gracilis, vastus medialis, vastus internus, biceps femoris, and semimembranosus. Mild involvements were observed in the sartorius and rectus femoris. Severe fatty infiltration was observed in the semitendinosus and vastus lateralis. In the calf muscles (Fig. 11), extensive fatty infiltration was found; however, the involvement of the posterior group of muscles (gastrocnemius and soleus) was more severe than that of the other muscles. This selective involvement was not observed in the edema of the calf muscles because edema with similar degrees was observed in each muscle of the calves.

Mild fatty infiltration of the calf muscles in proband 13 was found in the flexor digitorum longus and posterior group of muscles. Furthermore, muscle edema was also mild, which could be observed in the tibialis posterior, peroneus longus, and flexor digitorum longus. Furthermore, different degrees of fatty infiltration were observed in the thigh muscles of proband 15; however, the semitendinosus, sartorius, and gracilis were spared. Extensive edema of the thigh muscles was also observed in proband 15.

\section{Molecular analyses}

NGS analyses were performed using the samples obtained from all probands to establish a precise molecular diagnosis. After the screening of DNA sequence variants according to published protocols [14], a total of 38 genetic variants were identified, including those in the DYSF (13 variants), CAPN3 (seven variants), SGCG (four variants), SGCA (four variants), FKRP (three variants), $L M N A$ (three variants), SGCD (two variants), and TTN (two variants). Compound heterozygous variants were most common ( 15 of 24 probands, $62.50 \%$ ), followed by homozygous variants (6 of 24 probands, $25.00 \%$ ) and heterozygous variants ( 3 of 24 probands, 12.50\%). Heterozygous variants were only observed in three probands with LGMD 1B.

\section{Novel variants}

Eighteen of these variants were shown to be novel: c.714G > T (p.K238 N), c.5909C > T (p.P1970L), c.53 57G > A (p.W1786*), and c.3550C > T (p.Q1184*) in DYSF; c.468C > A (p.I156=), c.382G > T (p.D128Y), and c.753delC (p.I251Ifs"2) in CAPN3; c.702 + 1G > A, c.756 G > A (p.W252*), and c.477delG (p.V160Lfs*8) in SGCG; c.892delC (p.L298Cfs*23) and c.262delT (p.F88Sfs"123) in SGCA; c.1336G > T (p.D446Y) and c.431A > C (p.K14 $4 \mathrm{~T}$ ) in $L M N A$; c.503-2A $>\mathrm{G}$ and c.414delA (p.K138 $\mathrm{Nfs}^{* 4}$ ) in SGCD; and c.25006 $\mathrm{T}>\mathrm{C}$ (p.C8336R) and c.33938dupC (p.E11314Rfs*24) in TTN, while other identified variants have been reported earlier (Table 2) [31-43]. In family 6, in addition to two pathogenic variants, c.565C > G (p.L189 V) and c.4742G > A (p.R1581H) in DYSF were found to be single nucleotide polymorphisms (SNPs). The pathogenicity of the unreported synonymous variant in CAPN3, detected in proband 2, remains uncertain. Variants in genes related with EmeryDreifuss muscular dystrophy (EMD, LMNA, SYNE1, $S Y N E 2$, and FHL1) were not found in proband 12.

\section{Familial cosegregation analyses}

The blood samples collected from probands' parents were analyzed for familial cosegregation. Most familial cosegregations were verified, except for families 1 and 21 , owing to the death of the father and parents' refusal to offer samples, respectively. A homozygous variant c.1971_1973del (p.F658del) in CAPN3 was found in proband 1 with consanguineous parents. His mother was identified as a carrier; his late father is a suspected carrier; and the proband's brother (IV5; Fig. 2) was shown to have the same disease and to harbor the same homozygous variant. Furthermore, variants of proband 21 [c.151G > T (p.V51F), c.545A > G (p.Y182C)] were compound heterozygous, because her daughter was a carrier of the variant c.545A > G but not c.151G > T.

Homozygous variants were found in probands 1, 3, 4, 7,17 , and 23 , and definite consanguineous parents were determined in families 1,3 , and 7 (pedigrees shown in Fig. 2). In addition, several patients were determined in families $1,8,9,15$, and 21 according to similar clinical manifestations (pedigrees shown in Figs. 2, 3). The brother of proband 1 (IV5; Fig. 2) and the sister of proband 15 (II2; Fig. 3) had the same variants as the probands. The late brother of proband 1 (IV7), the sister of proband 8 (II6), the sister of proband 9 (II5), and the late sister of proband 21 (II1) were not analyzed by genetic testing (Figs. 2, 3). Other pedigrees are shown in Additional file 2.

De novo variants were determined in probands 18,19 , 20, and 22. Probands 18, 19, and 20 were diagnosed with LGMD 1B with autosomal dominant inheritance. Interestingly, the variant of proband 22 with LGMD $2 \mathrm{C}$ (c.477delG (p.V160Lfs"8) could not be detected in his parents, which indicated the possibility of spontaneous mutation of this variant. Considering that DNA samples of parents were from the blood, we could not exclude the possibility of chimeras because the variants might exist in other organs, including the gonads.

\section{Discussion}

In this study, we report a LGMD cohort in South China, wherein we identified deleterious variants in 23 out of 24 probands. Five clinical characteristics and three muscle MRI characteristics, which have rarely been 
Table 2 Genetic variants detected in the patients

\begin{tabular}{|c|c|c|c|c|c|}
\hline Mutant gene & Transcript ID & Family ID & Variant (Paternal) & Variant (Maternal) & Variant (De novo) \\
\hline \multirow[t]{7}{*}{ DYSF } & \multirow[t]{7}{*}{ NM_003494 } & Family 3 & \multicolumn{3}{|c|}{ c.863A > T (p.D288V) in homozygous state } \\
\hline & & Family 5 & c.799_800delTT (p.F267Lfs*5) & $\begin{array}{l}\text { c.680 T > C (p.1227T) / } \\
\text { c.714G > T (p.K238N) }\end{array}$ & \\
\hline & & Family 6 & c.5975delT (p.V1992Efs*20) & c.5909C > T (p.P1970L) & \\
\hline & & Family 8 & c.1375dupA (p.M459Nfs*15) & c.4200delC (p.I1401Sfs*47) & \\
\hline & & Family 13 & c.3988C > T (p.Q1330*) & c.1667 T > C (p.L556P) & \\
\hline & & Family 14 & c.1555G > A (p.G519R) & c.5357G > A (p.W1786*) & \\
\hline & & Family 16 & c.799_800delTT (p.F267Lfs*5) & c.3550C > T (p.Q1184*) & \\
\hline \multirow[t]{4}{*}{ CAPN3 } & \multirow[t]{4}{*}{ NM_000070 } & Family 1 & \multicolumn{3}{|c|}{ c.1971_1973del (p.F658del) in homozygous state ${ }^{b}$} \\
\hline & & Family 2 & $c .439 c>T\left(p . R 147^{*}\right)$ & $c .468 C>A(p .1156=)^{a}$ & \\
\hline & & Family 9 & c.1795dupA (p.T599Nfs*33) & $c .2050+1 G>A$ & \\
\hline & & Family 24 & $c .382 \mathrm{G}>\mathrm{T}(\mathrm{p} . \mathrm{D} 128 \mathrm{Y})$ & c.753delC (p.I251lfs*2) & \\
\hline \multirow[t]{3}{*}{ LMNA } & \multirow[t]{3}{*}{ NM_170707 } & Family 18 & & & c.1336G > T (p.D446Y) \\
\hline & & Family 19 & & & c.1357C > T (p.R453W) \\
\hline & & Family 20 & & & c.431A > C (p.K144T) \\
\hline \multirow[t]{3}{*}{ SGCG } & \multirow[t]{3}{*}{ NM_000231 } & Family 4 & \multicolumn{3}{|c|}{ c.768delC (p.S257Afs*23) in homozygous state } \\
\hline & & Family 7 & \multicolumn{3}{|c|}{ c.702 + 1G > A in homozygous state } \\
\hline & & Family 22 & & c.756G > A (p.W252*) & c.477delG (p.V160Lfs*8) \\
\hline \multirow[t]{2}{*}{ SGCA } & \multirow[t]{2}{*}{ NM_000023 } & Family 10 & c.292C > T (p.R98C) & c.892delC (p.L298Cfs*23) & \\
\hline & & Family 15 & c.262delT (p.F88Sfs*123) & c.409G > A (p.E137K) & \\
\hline \multirow[t]{2}{*}{$S G C D$} & \multirow[t]{2}{*}{ NM_000337 } & Family 17 & \multicolumn{3}{|c|}{ c.503-2A > G in homozygous state } \\
\hline & & Family 23 & \multicolumn{3}{|c|}{ c.414delA (p.K138Nfs*4) in homozygous state } \\
\hline \multirow[t]{2}{*}{ FKRP } & \multirow[t]{2}{*}{ NM_001039885 } & Family 11 & c.948delC (p.C317Afs*111) & c.545A > G (p.Y182C) & \\
\hline & & Family 21 & \multicolumn{3}{|c|}{ compound heterozygous variant $[\mathrm{c} .151 \mathrm{G}>\mathrm{T}(\mathrm{p} . \mathrm{V} 51 \mathrm{~F}), \mathrm{c.545 \textrm {A }}>\mathrm{G}(\mathrm{p} . \mathrm{Y} 182 \mathrm{C})]^{\mathrm{c}}$} \\
\hline TTN & NM_001267550 & Family 12 & c.25006 T > C (p.C8336R) & c.33938dupC (p.E11314Rfs*24) & \\
\hline
\end{tabular}

Bold variants are novel; the symbol * means the occurence of premature temination codon; the pathogenicity of variant ${ }^{\mathrm{a}}$ is uncertain Homozygous variant ${ }^{\mathrm{b}}$ was found in proband, and the proband's mother was a carrier. But proband's late father was undetermined

Compound heterozygous variant ${ }^{c}$ was found in proband, and the proband's daughter was a carrier (c.545A $>G$ ). But proband's parents refused to receive a genetic testing

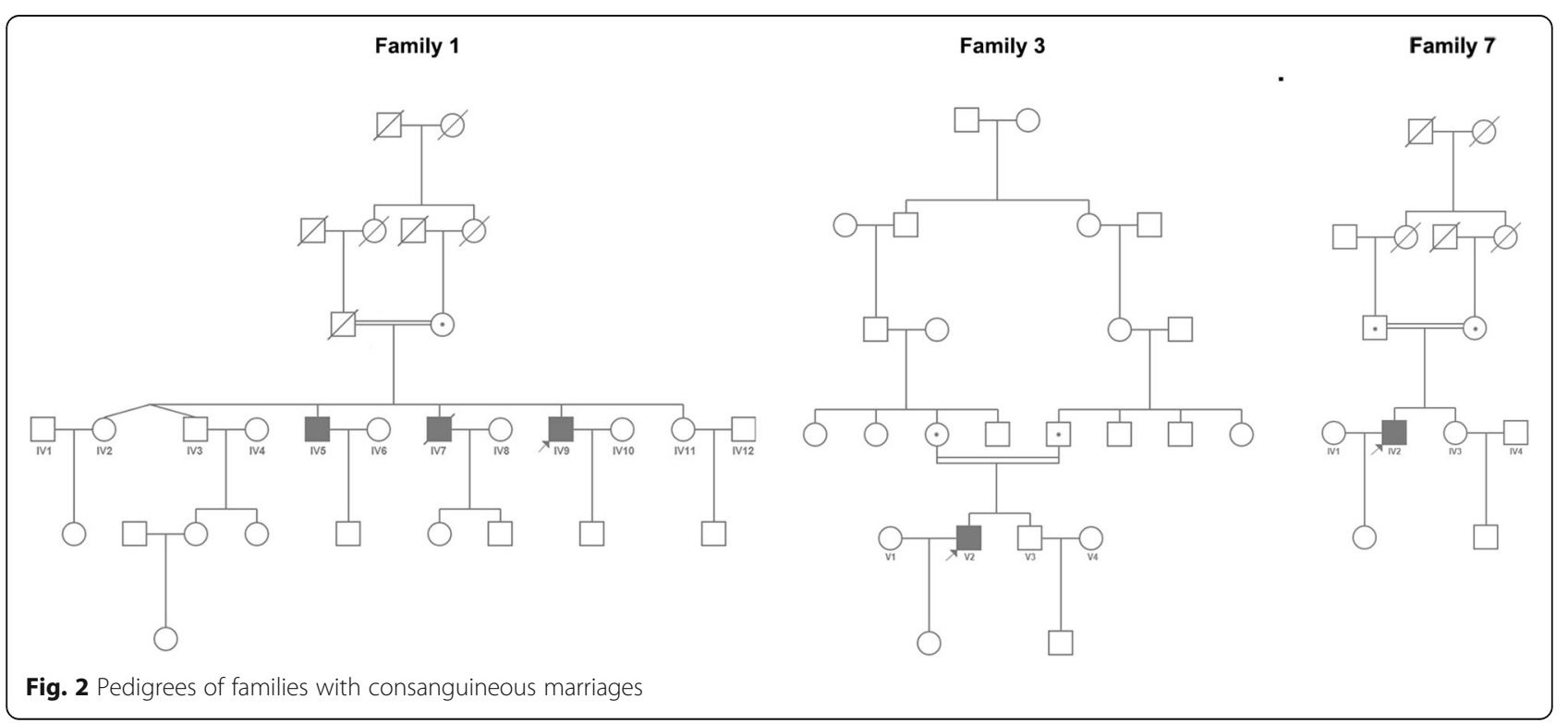




\section{Family 8}

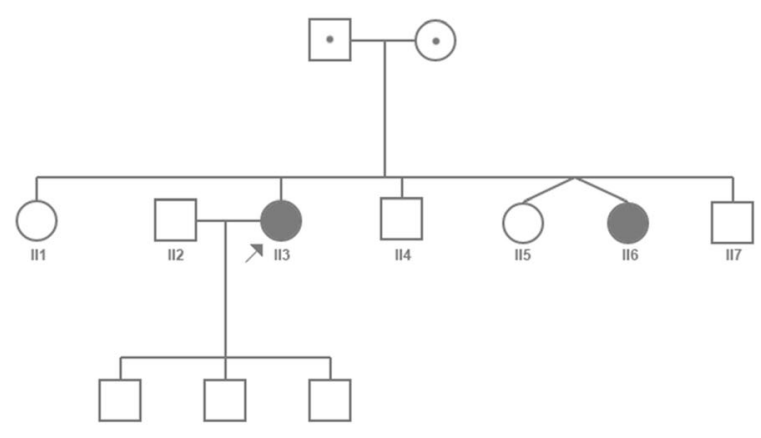

Family 9

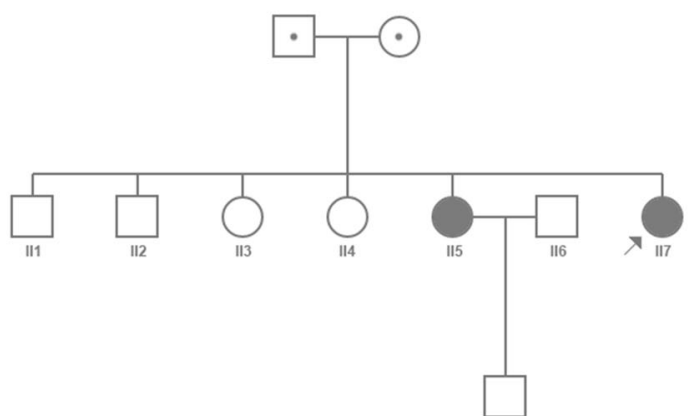

Family 15

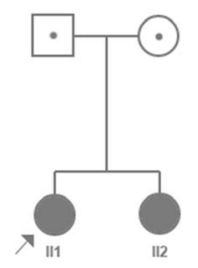

Family 21

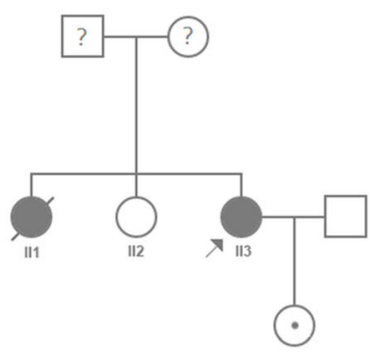

Fig. 3 Pedigrees of families with several patients

reported in the relevant subtypes, were observed. NGS analysis allowed the detection of 18 novel variants, and to the best of our knowledge, Chinese LGMD 2C patients have never been reported in the English literature thus far.

\section{The most common LGMD subtypes in China are LGMD} 2A, dysferlinopathy, sarcoglycanopathies, and LGMD 1B Previous LGMD cohorts in North China and Northeast China reported the most common LGMD subtypes to be dysferlinopathy $(49.52,38.46 \%)$ and LGMD 2A (24.76, 46.15\%), followed by sarcoglycanopathies (9.52, $7.69 \%)$ and LGMD 1B $(6.67,0 \%)[16,44]$. In the present cohort, these subtypes were also the top 4 , but the relative frequency of sarcoglycanopathies was obviously higher (26.67\%). Thus, dysferlinopathy is the most common LGMD subtype in the Chinese population, but the relative frequencies of sarcoglycanopathies and LGMD 2A differ between the southern and northern regions of China. Furthermore, although dysferlinopathy is also most frequent in Korean and Japanese populations, the frequencies of LGMD 2A, sarcoglycanopathies, and LGMD 1B vary among countries [32, 45]. Therefore, the top 4 LGMD subtypes in East Asia include dysferlinopathy, LGMD 2A, and LGMD 1B, but the ranks of LGMD 2A and LGMD 1B differ between regions. The frequency of sarcoglycanopathies varies greatly among regions, and the top 4 LGMD subtypes in Korea did not include sarcoglycanopathies [16, 32, 44, 45]. The reason for this phenomenon is not clear at present, which warrants further investigation.

Rare clinical features in LGMD 2F, 2l, 1B, dysferlinopathy, and the early onset recessive Emery-Dreifuss-like phenotype without cardiomyopathy

Most of the patients included in this study presented with the classical symptoms of LGMD; however, five rare characteristics were observed, which were hypermobility of the joints in proband 11 with LGMD 2I, proband 17 with LGMD 2F, and proband 18 with LGMD 1B; polydactyly in proband 14 with dysferlinopathy; patent foramen ovale in proband 16 with dysferlinopathy; delayed development of language in proband 23 with LGMD 2F; and atypical features in proband 12 with the early onset recessive Emery-Dreifuss-like phenotype without cardiomyopathy.

Hypermobility of the joints in the upper limbsobserved in some patients as well, although rare for LGMD 1B, 2F, and 2I patients-is a distinctive feature of 
congenital muscular disorders [46], and it should be carefully considered when diagnosing LGMD. Furthermore, proband 14 with dysferlinopathy presented with polydactyly in both hands. Polydactyly-related genes should be sequenced further for investigating the correlation between dysferlinopathy and polydactyly in proband 14. Proband 16 with dysferlinopathy had patent foramen ovale, which has never been reported in this condition. Although recurrent fat embolic strokes in a patient with Duchenne muscular dystrophy (DMD) with long bone fractures and a patent foramen ovale have been reported [47], whether such a patient should be prioritized to undergo surgery to close the defect over patients with simple patent foramen ovale needs to be assessed further because there is no evidence that patients with dysferlinopathy are more prone to bone fractures. Furthermore, although delayed language development has been reported in sarcoglycanopathies, this aspect has not been reported in patients with LGMD 2F [48, 49]. Because patients with DMD can present with delay in achieving both language and motor milestones [50] and manifestations of patients with sarcoglycanopathies are DMD-like [2], caution must be exercised to avoid the misdiagnosis of such patients with sarcoglycanopathies.

TTN gene variants were shown to cause titinopathy with various phenotypes, such as late-onset autosomal dominant tibial muscular dystrophy (MIM \#600334), LGMD 2 J (MIM \#608807), and hereditary myopathy with early respiratory failure (MIM \#603689) [51]. Recently, a novel phenotype (early onset recessive Emery-Dreifuss-like phenotype without cardiomyopathy) was proposed, together with the description of three families with this phenotype, including one Algerian and two French Caucasian families [52]. Five main clinical features of this phenotype were proposed, including (1) the coexistence of both limb-girdle weakness and early-onset contractures, preceding or accompanying the initial weakness; (2) early-onset muscular dystrophy with a normal neonatal period, proximal weakness in infancy and childhood, and a progressive course in adolescence and adulthood, with permanent loss of ambulation from age 13 to 36 years; (3) unaffected facial, bulbar, and oculomotor muscles; (4) high CK levels, decreasing in the later stages of the disease; and (5) no identified cardiomyopathy to date. The clinical presentation of proband 12 with the TTN gene variants agreed with these reported features, even if his manifestations were relatively mild due to the early stage of disease; however, some variations were observed. In previous studies, early contractures were reported to be observed in limbs, especially the elbows and ankles, and only one patient was reported to have the contracture of the cervical spine at the advanced stage [52]. However, our patient was shown to have contractures of the neck and ankle rather than the elbows. In addition, the malformations of the feet were prominent, including cavus feet and morton's toe in both feet. The CK level in this patient was less than twofold higher than the ULN, consistent with the mild weakness but unlike previously reported cases [52].

\section{Novel findings of muscle MRI features in LGMD 2A, dysferlinopathy, and the early onset recessive Emery- Dreifuss-like phenotype without cardiomyopathy}

Among eight probands with muscle MRI, three had dysferlinopathy (probands 3, 5, and 13); two had LGMD 2D (probands 10 and 15); and one each had LGMD 2A (proband 9), LGMD 2I (proband 11), and the early onset recessive Emery-Dreifuss-like phenotype without cardiomyopathy (proband 12). The features of muscle involvements in probands $3,10,11,13$, and 15 were consistent with previous reports [53-57]. Although different degrees of fatty infiltration of the posterior muscle group of the leg can be observed at the early stage of LGMD 2I $[55,56]$, proband 11 at the earliest stage of LGMD 2I was characterized only by mild fatty infiltration in the adductor magnus, which has also been suggested in previous studies [57]. Thus, LGMD 2I should still be considered in such patients.

Furthermore, the features of fatty infiltration are atypical in probands 5, 9, and 12 . Severe fatty infiltration of the thigh muscles observed in the MRI results obtained for probands 5 and 9 were consistent with severe motor dysfunctions as previously reported [55], but different muscle-sparing characteristics were observed in probands 5 and 9, who had advanced-stage LGMD. The MRI results obtained for proband 5, who had dysferlinopathy, showed that the adductor longus and gracilis were spared, unlike previously reported observations (the sartorius and gracilis were spared in advanced-stage dysferlinopathy) [13, 54], with the exception of a Chinese patient with dysferlinopathy reported to have similar muscle involvement [53]. In addition, the vastus lateralis, sartorius, and gracilis were shown to be commonly spared in the advanced stages of LGMD 2A, but proband 9 with LGMD 2A was shown to have the involvement of almost all muscles and a relatively mild involvement of the rectus femoris $[55,58]$. Therefore, muscle involvement may differ between the populations, indicating the need for further muscle MRI analyses of Chinese patients with LGMD.

The clinical manifestation of proband 12 with the early onset recessive Emery-Dreifuss-like phenotype without cardiomyopathy has been discussed in the text above, and the muscle MRI results were also distinguished from those of a previous report [52]. Severe fatty infiltration of the semitendinosus and vastus lateralis with relative sparing of other muscles in proband 12 differed from 
previous findings of diffuse and severe fatty infiltration in the posterior group of thigh muscles or all thigh muscles [52]. These different MRI features might be correlated with the stage of disease, because MRI scans were tested 6 years and 13-17 years after the onset of symptoms in our patients and three previously reported patients, respectively [52]. Moreover, although an obvious weakness in ankle dorsiflexion muscles rather than ankle plantarflexion muscles could be observed, the anterior group of calf muscles (responsible for ankle dorsiflexion) was shown to be affected more mildly than those of the posterior group (responsible for ankle plantarflexion) according to MRI results, which may represent a characteristic of the early stage of the early onset recessive Emery-Dreifuss-like phenotype without cardiomyopathy.

\section{Genetic analyses}

Our genetic analyses demonstrated that proband 6 has three variants in DYSF located in the same chromosome. The variant c.4742G $>$ A with a minor allele frequency (MAF) of 0.003582 has been reported as a polymorphism in east Asia [59], while the variant c.565C $>\mathrm{G}$ was reported to disrupt the exonic splicing enhancer and affect RNA splicing [60], but it is relatively frequent in east Asia (MAF $=0.007787$ ) and is considered benign/ likely benign according to the ClinVar database [26]. This led to the reconsideration of the pathogenicity of c.565C > G and its classification as a SNP. A missense variant (c.5909C $>$ T) with low MAF (not reported) was considered pathogenic. Patient 5 was found to carry two pathogenic variants in one chromosome (c.680 T > C; c.714G $>\mathrm{T}$ ) and one pathogenic variant in this gene in another chromosome (c.799_800del). Furthermore, c.680 $\mathrm{T}>\mathrm{C}$ was identified in a patient with dysferlinopathy [31], and both c.680 T $>\mathrm{C}$ and c.714G $>\mathrm{T}$ can be classified as pathogenic according to the ACMG guidelines [20]. Although it is unclear whether one of these variants is more pathogenic or not, it is not a crucial issue that will affect reproductive counselling, because, owing to their very small genetic distance, they are tightly linked [61].

The variant $(\mathrm{c} .545 \mathrm{~A}>\mathrm{G})$ in $F K R P$ has been reported to be a founder variant in a North China LGMD cohort [43], and the results of our study supported this theory. Besides, a LGMD 2I cohort from the Taiwan region indicated that half of the patients had the variant (c.545A > G) [39]. Thus, the variant (c.545A $>$ G) might be a founder variant in China. The data of the variant spectrum of LGMD 2I in Korea and Japan are necessary to determine whether the variant $($ c. $545 \mathrm{~A}>\mathrm{G})$ was the hot spot variant in East Asia; this aspect warrants further investigation. Furthermore, proband 22 with LGMD $2 \mathrm{C}$ had compound heterozygous variants, one of which (c.756G > A) was inherited from his mother; however, another one (c.477delG) was not detected in his parents' DNA samples extracted from blood cells. There are two possibilities to explain this phenomenon: (1) the de novo variant originated from spontaneous mutations during spermatogenesis or early development of the embryo, or (2) the father is a chimera: the variant does not exist in blood cells but might be present in other organs, including the gonads. If the father is a chimera with the variant (c. 477delG) in his testes, his future offspring would have a chance of being affected by the disease. As a next step, therefore, the father's semen should be collected for gene sequencing to determine whether the variant exists in the gonads.

\section{Conclusions}

In conclusion, this study described a LGMD cohort of 30 Chinese patients in 24 families carrying a total of 38 different variants. The frequencies of LGMD subtypes were discussed. Furthermore, five rare clinical characteristics and three rare muscle MRI characteristics were reported. These distinct clinical phenotypes and newly identified genotypes of patients in our LGMD cohort contributed to the novel recognitions of the clinical and genetic spectrums of LGMD, which may further promote a comprehensive understanding of the phenotypegenotype correlation and help improve the diagnosis and treatment of these diseases, as well as reproductive counselling.

\section{Additional files}

Additional file 1: NGS protocol and the list of genes in the neuromuscular disorder panel. (DOCX $20 \mathrm{~kb}$ )

Additional file 2: Pedigrees of families with only one patient. (TIF 112 kb)

\section{Abbreviations}

ACMG: American College of Medical Genetics and Genomics; CK: Creatine kinase; DMD: Duchenne muscular dystrophy; EMG: Electromyography;

IHC: Immunohistochemical staining; LGMD: Limb-girdle muscular dystrophy; MAF: Minor allele frequency; MRC: Medical Research Council; MRI: Magnetic resonance imaging; NGS: Next-generation sequencing; SNP: Single nucleotide polymorphism; ULN: Upper normal limit

\section{Acknowledgements}

We would like to thank people who offered technical or editing support and all patients for participating in this study.

\section{Funding}

This study was supported by the Natural Science Foundation of China (grant nos. 81771359, 81471280, and 81271401); the Guangdong Provincial Science and Technology Plan (grant no. 2014A020212130); the Guangzhou City Science and Technology Plan (grant nos. 1561000153/201508020012); the National Key Clinical Department and Key Discipline of Neurology, the Guangdong Provincial Key Laboratory for Diagnosis and Treatment of Major Neurological Diseases (no. 2014B030301035); the Southern China International Cooperation Base for Early Intervention and Functional Rehabilitation of Neurological Diseases (no. 2015B050501003); Guangdong Provincial Engineering Center for Major Neurological Disease Treatment, Science and Technology Planning Project of Guangzhou (no. 201604020010); 
and Major Medical Collaboration and Innovation Program of Guangzhou Science Technology and Innovation Commission (grant no. 201604020020).

\section{Availability of data and materials}

The datasets used and/or analyzed during this study are available from the corresponding author upon request.

\section{Authors' contributions}

LW and CZ designed this study. Clinical information was provided by CZ and LW. All authors contributed to data collection. LW, SL, and HL organized and analyzed the data. LW wrote the manuscript. WWZ and CZ made revisions. CZ supervised the project. All authors read and approved the final manuscript.

\section{Ethics approval and consent to participate}

This study and all protocols used conformed to the ethical guidelines of the 1975 Declaration of Helsinki, as reflected in an a priori approval by the Human Research Committee of the Hereditary Neurological Disease Clinics of The First Affiliated Hospital, Sun Yat-sen University. Informed consent was obtained from each patient included in the study.

\section{Consent for publication}

The adult patients and the parents of the children described in this article provided consent for participation in the study and for publishing the obtained results,

\section{Competing interests}

The authors declare that they have no competing interests.

\section{Publisher's Note}

Springer Nature remains neutral with regard to jurisdictional claims in published maps and institutional affiliations.

\section{Author details}

'Department of Neurology, National Key Clinical Department and Key Discipline of Neurology, The First Affiliated Hospital, Sun Yat-sen University, 58 Zhongshan 2 Road, Guangzhou 510080, GD, China. ${ }^{2}$ Department of Molecular and Human Genetics, Baylor College of Medicine, Houston, TX 77030, USA. ${ }^{3}$ AmCare Genomics Lab, Guangzhou 510300, GD, China. ${ }^{4}$ Department of Health Care, The First Affiliated Hospital, Sun Yat-sen University, Guangzhou 510080, GD, China.

Received: 1 March 2018 Accepted: 27 June 2018

\section{Published online: 14 August 2018}

\section{References}

1. Mah JK, Korngut L, Fiest KM, Dykeman J, Day L, Pringsheim T, et al. A systematic review and meta-analysis on the epidemiology of the muscular dystrophies. Can J Neurol Sci. 2016;43:163-77.

2. Liewluck T, Milone M. Untangling the complexity of limb-girdle muscular dystrophies. Muscle Nerve. 2018;[Epub ahead of print]. https://doi.org/10. 1002/mus.26077.

3. Deenen JC, Horlings CG, Verschuuren JJ, Verbeek AL, van Engelen BG. The epidemiology of neuromuscular disorders: a comprehensive overview of the literature. J Neuromuscul Dis. 2015;2:73-85.

4. Mah JK, Korngut L, Dykeman J, Day L, Pringsheim T, Jette N. A systematic review and meta-analysis on the epidemiology of Duchenne and Becker muscular dystrophy. Neuromuscul Disord. 2014;24:482-91.

5. Magri F, Nigro V, Angelini C, Mongini T, Mora M, Moroni I, et al. The italian limb girdle muscular dystrophy registry: relative frequency, clinical features, and differential diagnosis. Muscle Nerve. 2017;55:55-68.

6. Sveen ML, Schwartz M, Vissing J. High prevalence and phenotype-genotype correlations of limb girdle muscular dystrophy type 21 in Denmark. Ann Neurol. 2006:59:808-15.

7. Bastian A, Mageriu V, Micu G, Manole E. The growing family of limb-girdle muscular dystrophies: old and newly identified members. Rom J Intern Med. 2015;53:13-24.

8. Khadilkar SV, Faldu HD, Patil SB, Singh R. Limb-girdle muscular dystrophies in India: a review. Ann Indian Acad Neurol. 2017;20:87-95.

9. Vissing J. Limb girdle muscular dystrophies: classification, clinical spectrum and emerging therapies. Curr Opin Neurol. 2016;29:635-41.
10. Straub V, Bertoli M. Where do we stand in trial readiness for autosomal recessive limb girdle muscular dystrophies? Neuromuscul Disord. 2016;26: 111-25.

11. Angelini C, Fanin M. Limb girdle muscular dystrophies: clinical-genetical diagnostic update and prospects for therapy. Expert Opinion on Orphan Drugs. 2017;5:769-84.

12. Thompson R, Straub V. Limb-girdle muscular dystrophies - international collaborations for translational research. Nat Rev Neurol. 2016;12:294-309.

13. Paradas C, Llauger J, Diaz-Manera J, Rojas-García R, De Luna N, Iturriaga C, et al. Redefining dysferlinopathy phenotypes based on clinical findings and muscle imaging studies. Neurology. 2010;75:316-23.

14. Tian X, Liang WC, Feng Y, Wang J, Zhang W, Chou CH, et al. Expanding genotype/phenotype of neuromuscular diseases by comprehensive target capture/NGS. Neurol Genet. 2015;1:e14.

15. Stark Z, Schofield D, Alam K, Wilson W, Mupfeki N, Macciocca I, et al. Prospective comparison of the cost-effectiveness of clinical whole-exome sequencing with that of usual care overwhelmingly supports early use and reimbursement. Genet Med. 2017;19:867-74.

16. $Y u$ M, Zheng $Y$, Jin $S$, Gang $Q$, Wang $Q$, Yu P, et al. Mutational spectrum of Chinese LGMD patients by targeted next-generation sequencing. PLoS One. 2017;12:e0175343.

17. Khadilkar SV, Patel BA, Lalkaka JA. Making sense of the clinical spectrum of limb girdle muscular dystrophies. Pract Neurol. 2018;18(3):201-10. https:// doi.org/10.1136/practneurol-2017-001799.

18. Bing Q, Hu K, Tian Q, Zhao Z, Shen H, Li N, et al. Semi-quantitative assessment of lower limb MRI in dystrophinopathy. Int J Clin Exp Med. 2016; 9:13723-32.

19. Meola G, Bugiardini E, Cardani R. Muscle biopsy. J Neurol. 2012;259:601-10.

20. Spuler S, Carl M, Zabojszcza J, Straub V, Bushby K, Moore SA, et al. Dysferlindeficient muscular dystrophy features amyloidosis. Ann Neurol. 2008;63:3238.

21. 1000 Genomes Project. http://www.internationalgenome.org/1000genomes-browsers. Accessed 26 Apr 2018.

22. Exome Aggregation Consortium. http://exac.broadinstitute.org. Accessed 26 Apr 2018

23. PolyPhen-2. http://genetics.bwh.harvard.edu/pph2/. Accessed 26 Apr 2018.

24. SIFT. http://sift.jcvi.org/. Accessed 26 Apr 2018.

25. MutationTaster. http://www.mutationtaster.org/. Accessed 26 Apr 2018.

26. ClinVar. https://www.ncbi.nlm.nih.gov/clinvar/. Accessed 26 Apr 2018.

27. Human Gene Mutation Database (HGMD). http://www.hgmd.cf.ac.uk/ac/ index.php. Accessed 26 Apr 2018.

28. Leiden Open Variation Database (LOVD). http://www.lovd.nl/3.0/home. Accessed 26 Apr 2018

29. Google Scholar. http://scholar.google.com.hk/. Accessed 26 Apr 2018.

30. Richards S, Aziz N, Bale S, Bick D, Das S, Gastier-Foster J, et al. Standards and guidelines for the interpretation of sequence variants: a joint consensus recommendation of the American College of Medical Genetics and Genomics and the Association for Molecular Pathology. Genet Med. 2015 17:405-24.

31. Jin SQ, Yu M, Zhang W, Lyu H, Yuan Y, Wang ZX. Dysferlin gene mutation spectrum in a large cohort of Chinese patients with dysferlinopathy. Chin Med J (Engl). 2016;129:2287-93

32. Seong MW, Cho A, Park HW, Seo SH, Lim BC, Seol D, et al. Clinical applications of next-generation sequencing-based gene panel in patients with muscular dystrophy: Korean experience. Clin Genet. 2016:89:484-8.

33. Kawai H, Akaike M, Kunishige M, Inui T, Adachi K, Kimura C, et al. Clinical, pathological, and genetic features of limb-girdle muscular dystrophy type $2 \mathrm{~A}$ with new calpain 3 gene mutations in seven patients from three Japanese families. Muscle Nerve. 1998;21:1493-501.

34. Chae J, Minami N, Jin Y, Nakagawa M, Murayama K, Igarashi F, et al. Calpain 3 gene mutations: genetic and clinico-pathologic findings in limb-girdle muscular dystrophy. Neuromuscul Disord. 2001:11:547-55.

35. Park HJ, Jang H, Lee JH, Shin HY, Cho SR, Park KD, et al. Clinical and pathological heterogeneity of Korean patients with CAPN3 mutations. Yonsei Med J. 2016;57:173-9.

36. Babameto-Laku A, Tabaku M, Tashko V, Cikuli M, Mokini V. The first case of primary alpha-sarcoglycanopathy identified in Albania, in two siblings with homozygous alpha-sarcoglycan mutation. Genet Couns. 2011:22:377-83.

37. Ljunggren A, Duggan D, McNally E, Boylan KB, Gama CH, Kunkel LM, et al. Primary adhalin deficiency as a cause of muscular dystrophy in patients with normal dystrophin. Ann Neurol. 1995;38:367-72. 
38. Duggan DJ, Gorospe JR, Fanin M, Hoffman EP, Angelini C. Mutations in the sarcoglycan genes in patients with myopathy. N Engl J Med. 1997;336:618-24

39. Liang WC, Hayashi YK, Ogawa M, Wang CH, Huang WT, Nishino I, et al. Limb-girdle muscular dystrophy type 21 is not rare in Taiwan. Neuromuscul Disord. 2013;23:675-81.

40. Krahn M, Béroud C, Labelle V, Nguyen K, Bernard R, Bassez G, et al. Analysis of the DYSF mutational spectrum in a large cohort of patients. Human Mutat. 2009:30:E345-75.

41. Kergourlay V, Raï G, Blandin G, Salgado D, Béroud C, Lévy N, et al. Identification of splicing defects caused by mutations in the dysferlin gene. Hum Mutat. 2014;35:1532-41.

42. Punetha J, Kesari A, Uapinyoying P, Giri M, Clarke NF, Waddell LB, et al. Targeted re-sequencing emulsion PCR panel for myopathies: results in 94 cases. J Neuromuscul Dis. 2016;27:209-25.

43. Fu $X$, Yang $H$, Wei $C$, Jiao $H$, Wang $S$, Yang $Y$, et al. FKRP mutations, including a founder mutation, cause phenotype variability in Chinese patients with dystroglycanopathies. J Hum Genet. 2016;61:1013-20.

44. Mahmood OA, Jiang X, Zhang Q. Limb-girdle muscular dystrophy subtypes: first-reported cohort from northeastern China. Neural Regen Res. 2013;8:1907-18.

45. Nishikawa A, Mitsuhashi S, Miyata N, Nishino I. Targeted massively parallel sequencing and histological assessment of skeletal muscles for the molecular diagnosis of inherited muscle disorders. J Med Genet. 2017;54:104-10.

46. Voermans NC, Bonnemann CG, Hamel BCJ, Jungbluth H, van Engelen BG. Joint hypermobility as a distinctive feature in the differential diagnosis of myopathies. J Neurol. 2009;256:13-27.

47. Bugnitz CJ, Cripe LH, Lo WD, Flanigan KM. Recurrent fat embolic strokes in a patient with Duchenne muscular dystrophy with long bone fractures and a patent foramen Ovale. Pediatr Neurol. 2016;63:76-9.

48. Duncan DR, Kang PB, Rabbat JC, Briggs CE, Lidov HG, Darras BT, et al. A novel mutation in two families with limb-girdle muscular dystrophy type 2C. Neurology. 2006;67:167-9.

49. Kapoor S, Tatke M, Aggarwal S, Gupta A. Beta-Sarcoglycanopathy. Indian J Pediatr. 2005;72:71-4.

50. Cyrulnik SE, Fee RJ, De Vivo DC, Goldstein E, Hinton VJ. Delayed developmental language milestones in children with Duchenne's muscular dystrophy. J Pediatr. 2007;150:474-8.

51. Hackman P, Udd B, Bonnemann CG, Ferreiro A, Titinopathy Database Consortium. 219th ENMC International Workshop Titinopathies International database of titin mutations and phenotypes, Heemskerk, The Netherlands, 29 April-1 May 2016. Neuromuscul Disord. 2017;27:396-407.

52. De Cid R, Ben Yaou R, Roudaut C, Charton K, Baulande S, Leturcq F, et al. A new titinopathy: childhood-juvenile onset Emery-Dreifuss-like phenotype without cardiomyopathy. Neurology. 2015;85:2126-35.

53. Jin S, Du J, Wang Z, Zhang W, Lv H, Meng L, et al. Heterogeneous characteristics of MRI changes of thigh muscles in patients with dysferlinopathy. Muscle Nerve. 2016;54:1072-9.

54. Kesper K, Kornblum C, Reimann J, Lutterbey G, Schroder R, Wattjes MP. Pattern of skeletal muscle involvement in primary dysferlinopathies: a whole-body 3.0-T magnetic resonance imaging study. Acta Neurol Scand. 2009;120:111-8

55. ten Dam L, van der Kooi AJ, van Wattingen $M$, de Haan RJ, de Visser $M$. Reliability and accuracy of skeletal muscle imaging in limb-girdle muscular dystrophies. Neurology. 2012;79:1716-23.

56. Fischer D, Walter MC, Kesper K, Petersen JA, Aurino S, Nigro V, et al. Diagnostic value of muscle MRI in differentiating LGMD2I from other LGMDs. J Neurol. 2005;252:538-47.

57. Willis TA, Hollingsworth KG, Coombs A, Sveen ML, Andersen S, Stojkovic T, et al. Quantitative magnetic resonance imaging in limb-girdle muscular dystrophy 2l: a multinational cross-sectional study. PLoS One. 2014;9:e90377.

58. Mercuri E, Pichiecchio A, Allsop J, Messina S, Pane M, Muntoni F. Muscle MR in inherited neuromuscular disorders: past, present, and future. J Magn Reson Imaging. 2007;25:433-40

59. Takahashi T, Aoki M, Tateyama M, Kondo E, Mizuno T, Onodera Y, et al. Dysferlin mutations in Japanese Miyoshi myopathy: relationship to phenotype. Neurology. 2003:60:1799-804.

60. Kergourlay V, Rai G, Blandin G, Salgado D, Béroud C, Lévy N, et al. Identification of splicing defects caused by mutations in the dysferlin gene. Hum Mutat. 2014;35:1532-41.

61. Allen-Brady K, Camp NJ. Genetic distance and markers used in linkage mapping. Methods Mol Biol. 2011;713:43-53.

\section{Ready to submit your research? Choose BMC and benefit from:}

- fast, convenient online submission

- thorough peer review by experienced researchers in your field

- rapid publication on acceptance

- support for research data, including large and complex data types

- gold Open Access which fosters wider collaboration and increased citations

- maximum visibility for your research: over $100 \mathrm{M}$ website views per year

At BMC, research is always in progress.

Learn more biomedcentral.com/submissions 